Umberto Vitolo, Annalisa Chiappella, Ileana Baldi, Domenico Novero, Lorella Orsucci, Umberto Ricardi, San Giovann Battista Hospital and University, Turin; Andrés J.M. Ferreri, San Raffaele Scientific Institute; Monica Balzarotti, Humanitas Institute, Rozzano (Milan): Giovanni Martinelli, European Institute of Oncology, Milan; Maurizio Martelli, University La Sapienza of Rome, Rome Chiara Bottelli, Spedali Civil Hospital, Brescia; Annarita Conconi, Hospital Maggiore della Carità, "Amedeo Avogadro" University of Eastern Piedmont, Novara; Francesco Merli, Maria Nuova Hospital, Reggio Emilia;

Vincenzo Pavone, University and Hospital, Bari; Sergio Storti, University Cattolica del Sacro Cuore, Campobasso Italy; Henry Gomez, Instituto Nacional de Enfermedades Neoplásicas, Lima,

Peru; Armando Lopez-Guillermo, Hospital Clínic, Institut d'Investigacions

Biomèdiques August Pi i Sunyer, BarceIona, Spain; Mary K. Gospodarowicz, Princess Margaret Hospital, Toronto, Ontario, Canada; Franco Cavalli and

Emanuele Zucca, Oncology Institute of Southern Switzerland, San Giovanni Hospital, Bellinzona, Switzerland; and Andreas H. Sarris, Hygeia Hospital Marousi, Athens, Greece.

Submitted July 7, 2010; accepted March 28, 2011; published online ahead of print at www.jco.org on June 6, 2011

A.H.S. and E.Z. were co-senior authors. Authors' disclosures of potential conflicts of interest and author contributions are found at the end of this article.

Clinical Trials repository link available on JCO.org.

Corresponding author: Umberto Vitolo, MD, SC Ematologia 2, Azienda Ospedaliera S. Giovanni Battista, Corso Bramante 8810126 Torino, Italy; e-mail uvitolo@molinette.piemonte.it.

() 2011 by American Society of Clinical Oncology

0732-183X/11/2920-2766/\$20.00

DOI: 10.1200/JCO.2010.31.4187

\title{
First-Line Treatment for Primary Testicular Diffuse Large B-Cell Lymphoma With Rituximab-CHOP, CNS Prophylaxis, and Contralateral Testis Irradiation: Final Results of an International Phase II Trial
}

Umberto Vitolo, Annalisa Chiappella, Andrés J.M. Ferreri, Maurizio Martelli, Ileana Baldi, Monica Balzarotti, Chiara Bottelli, Annarita Conconi, Henry Gomez, Armando Lopez-Guillermo, Giovanni Martinelli, Francesco Merli, Domenico Novero, Lorella Orsucci, Vincenzo Pavone, Umberto Ricardi, Sergio Storti, Mary K. Gospodarowicz, Franco Cavalli, Andreas H. Sarris, and Emanuele Zucca

\section{$\begin{array}{llllllll}\text { A } & \text { B } & \mathbf{S} & \mathbf{T} & \mathbf{R} & \mathbf{A} & \mathbf{C} & \mathbf{T}\end{array}$}

\section{Purpose}

Primary testicular lymphoma (PTL) has poor prognosis with failures in contralateral testis, CNS, and extranodal sites. To prevent these events, we designed an international phase II trial (International Extranodal Lymphoma Study Group 10 [IELSG-10]) that addressed feasibility and activity of conventional chemoimmunotherapy associated with CNS prophylaxis and contralateral testis irradiation. The trial was conducted by the IELSG and the Italian Lymphoma Foundation.

\section{Patients and Methods}

Fifty-three patients (age 22 to 79 years) with untreated stage I or II PTL were treated with six to eight courses of rituximab added to cyclophosphamide, doxorubicin, vincristine, and prednisone (R-CHOP) every 21 days (R-CHOP21); four doses of intrathecal methotrexate (IT-MTX) and radiotherapy $(\mathrm{RT})$ to the contralateral testis (30 Gy) for all patients and to regional lymph nodes (30 to $36 \mathrm{~Gy}$ ) for stage II disease.

\section{Results}

All patients received $\mathrm{R}-\mathrm{CHOP} 21,50$ received CNS prophylaxis, and 47 received testicular $\mathrm{RT}$. With a median follow-up of 65 months, 5-year progression-free survival and overall survival rates were $74 \%(95 \% \mathrm{Cl}, 59 \%$ to $84 \%)$ and $85 \%(95 \% \mathrm{Cl}, 71 \%$ to $92 \%)$, respectively. Ten patients relapsed or progressed: two in lymph nodes, five in extranodal organs, and three in the CNS. The 5-year cumulative incidence of CNS relapse was $6 \%(95 \% \mathrm{Cl}, 0 \%$ to $12 \%)$. No contralateral testis relapses occurred. Ten patients died: Iymphoma $(n=6)$, secondary leukemia $(n=2)$, heart failure $(n=1)$, and gastric cancer $(n=1)$. Grade 3 to 4 toxicities were neutropenia, $28 \%$; infections, $4 \%$; and neurologic, $13 \%$. No deaths occurred as a result of toxicity.

\section{Conclusion}

This international prospective trial shows that combined treatment with R-CHOP21, IT-MTX, and testicular RT was associated with a good outcome in patients with PTL. RT avoided contralateral testis relapses, but CNS prophylaxis deserves further investigation.

\section{J Clin Oncol 29:2766-2772. (C) 2011 by American Society of Clinical Oncology}

\section{INTRODUCTION}

Primary testicular non-Hodgkin's lymphoma (PTL) is an uncommon disease and accounts for less than $5 \%$ of testicular malignancies and $1 \%$ to $2 \%$ of nonHodgkin's lymphomas. ${ }^{1-5}$ Most patients present with localized stages $\mathrm{I}$ to $\mathrm{II}_{\mathrm{E}}$ disease; nevertheless, the outcome is poor. ${ }^{1,2,6}$ The two largest series of PTL reported so far are a retrospective survey of 373 patients $^{1}$ by the International Extranodal Lymphoma Study Group (IELSG) and a US populationbased study of 769 patients in the Surveillance,
Epidemiology and End Results (SEER) database. ${ }^{4}$ Both studies showed a median overall survival (OS) of 4 to 5 years and a continuous risk of relapse and disease-related deaths, even 10 years after diagnosis. ${ }^{1,4}$ Distant relapses at extranodal sites, especially the CNS and the contralateral testis, remain the most therapeutic challenges in PTL, even in patients who receive doxorubicin-based chemotherapy. ${ }^{1,6-10}$ Evidence from retrospective reports ${ }^{1,4,11,12}$ suggests that radiotherapy (RT) to the contralateral testis could reduce the risk of treatment failure at this site. CNS relapses, in both brain parenchyma and 
meninges, remain a major problem since they are definitely more common than in other aggressive lymphomas, and with 5- and 10year risks of $20 \%$ and $35 \%$, they constitute a dismal event. ${ }^{1,7}$ Importantly, the best strategy to prevent CNS relapse remains to be defined. The addition of rituximab to cyclophosphamide, doxorubicin, vincristine, and prednisone every 21 days (R-CHOP21) or dosedense CHOP every 14 days (CHOP14) significantly improves OS and progression-free survival (PFS) compared with $\mathrm{CHOP}$ alone in either elderly or young low-risk patients with diffuse large B-cell lymphoma (DLBCL). ${ }^{13-15}$ However, no specific data are available for PTL patients.

Herein, we report the final result of what is, to the best of our knowledge, the first prospective trial in PTL (IELSG-10), which addressed the safety and efficacy of a combined treatment strategy that included R-CHOP21 and both CNS and testicular prophylaxis. This trial was designed to prospectively evaluate the treatment recommendations that could be drawn from the previous IELSG retrospective study, ${ }^{1}$ and it provides a contribution to defining a proper treatment for PTL.

\section{PATIENTS AND METHODS}

\section{Study Design}

The IELSG-10 trial was a multicenter phase II prospective study aimed at evaluating the PFS of the R-CHOP21 regimen in combination with intrathecal methotrexate (IT-MTX) and locoregional RT for the treatment of PTL. The study was designed by the IELSG and conducted in collaboration with the Italian Lymphoma Foundation. The protocol was approved by the institutional review boards and/or ethics committees of each participating institution. The study was conducted in accordance with the Helsinki declaration. All patients gave written informed consent.

\section{Patient Selection and Assessment}

Criteria for eligibility were previously untreated PTL with DLBCL histology, age $\geq 18$ years, and Ann Arbor stage I or II (Fig 1). Bilateral testicular involvement at presentation was considered a single extranodal site, and those without nodal involvement were classified as stage I. Exclusion criteria were Eastern Cooperative Oncology Group (ECOG) performance status $\geq 2$, major organ dysfunction, HIV seropositivity, malignancies within the last 5 years, poor cardiac function (left ventricular ejection fraction $<50 \%$ ), and CNS involvement at diagnosis.

Histologic diagnosis was centrally reviewed by D.N. according to the WHO classification. ${ }^{16}$ Baseline assessment included bone marrow biopsy; full laboratory workup; HIV serology; testicular ultrasound; chest, abdomen, and pelvis computed tomography scans; multiple-gated acquisition scan or echocardiography; and lumbar puncture with cytologic examination of the cerebrospinal fluid.

\section{Treatment Plan}

The trial design is shown in Figure 2. All patients had diagnostic orchiectomy. The treatment consisted of a standard dose of R-CHOP21, which included $375 \mathrm{mg} / \mathrm{m}^{2}$ rituximab day 0 or day $1,750 \mathrm{mg} / \mathrm{m}^{2}$ cyclophosphamide day $1,50 \mathrm{mg} / \mathrm{m}^{2}$ doxorubicin day $1,1.4 \mathrm{mg} / \mathrm{m}^{2}$ (maximum $2 \mathrm{mg}$ ) vincristine day 1 , and $100 \mathrm{mg}$ prednisone day 1 to 5 . All patients were restaged after three courses of R-CHOP21. Stage I patients received six courses of R-CHOP21. Stage II patients received a total of six or eight courses of R-CHOP21 if they had a complete response (CR) or partial response (PR) after the third course. Chemoimmunotherapy was delivered in an outpatient setting.

All patients were planned to receive CNS prophylaxis with IT-MTX, 12 mg total dose, weekly for four times, during the first two R-CHOP21 courses. At the end of chemoimmunotherapy, prophylactic irradiation to the contralateral testis at 25 to $30 \mathrm{~Gy}$ was delivered to all patients. Patients with stage II disease at diagnosis, beyond prophylactic testicular RT, received involved field RT (IF-RT), which included the entire involved lymph node region with or without an adjacent lymph node region. Minimum IF-RT, for patients with limited para-aortic lymph node involvement only, includes the centimeterwide field; maximum IF includes the inverted Y field with para-aortic Iymph

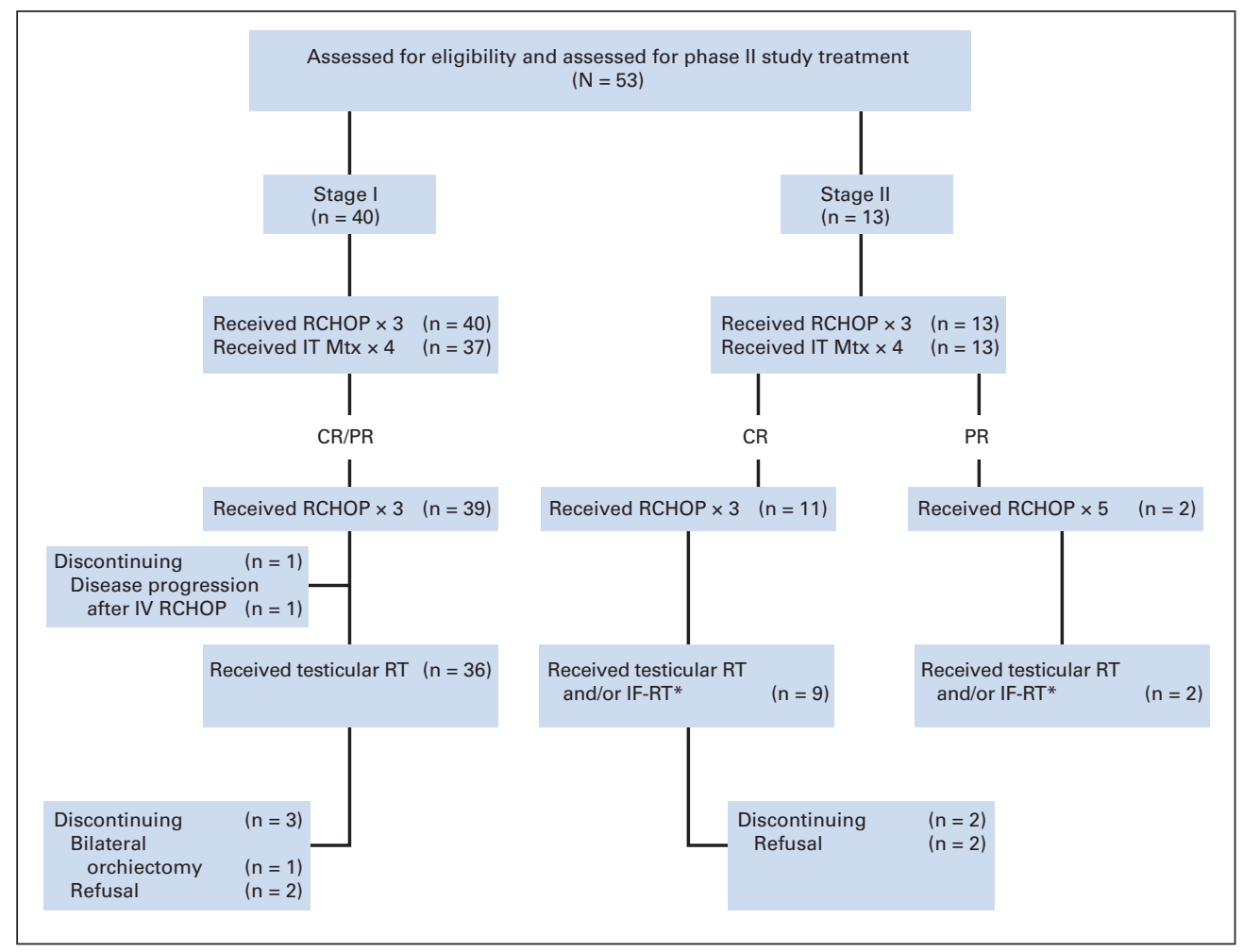

Fig 1. CONSORT diagram. Flow of the 53 patients enrolled onto the International Extranodal Lymphoma Study Group 10 (IELSG-10) study. ${ }^{*}$ ) Details on radiotherapy (RT) are reported in section on feasibility. In stage II patients, 11 received prophylactic testicular RT and nine received retroperitoneal lymph-node RT. CR, complete response; IF-RT, involved field radiotherapy; IT-Mtx, intrathecal methotrexate; IV, intravenous; PR, partial response; RCHOP, rituximab plus cyclophosphamide, doxorubicin, vincristine, and prednisone. 


\begin{tabular}{|c|c|c|}
\hline Characteristic & No. of Patients & $\%$ \\
\hline \multicolumn{3}{|l|}{ Age, years } \\
\hline Median & \multicolumn{2}{|l|}{64} \\
\hline Range & \multicolumn{2}{|l|}{$22-79$} \\
\hline \multicolumn{3}{|l|}{ Ann Arbor stage } \\
\hline$I_{E}$ & 40 & 75 \\
\hline$\|_{E}$ & 13 & 25 \\
\hline Bilateral testicular involvement & 4 & 8 \\
\hline Lymph node involvement & 13 & 25 \\
\hline \multicolumn{3}{|l|}{ ECOG performance status } \\
\hline 0 & 47 & 89 \\
\hline 1 & 4 & 7 \\
\hline 2 & 2 & 4 \\
\hline B symptoms & 2 & 4 \\
\hline Elevated LDH serum level* & 6 & 12 \\
\hline Elevated $\beta_{2}$-microglobulin serum levelt & 6 & 14 \\
\hline $\begin{array}{l}\text { Abbreviations: ECOG, Eastern Coope } \\
\text { tate dehydrogenase. } \\
\text { *Assessed in } 50 \text { patients; three missing } \\
\text { †Assessed in } 42 \text { patients; } 11 \text { missing. }\end{array}$ & Oncology Group; & \\
\hline
\end{tabular}

node region and bilateral pelvic lymph nodes. Patients in $\mathrm{CR}$ after R-CHOP21 received IF-RT at 30 to 35 Gy; IF-RT at 35 to 45 Gy was administered to those in $\mathrm{CR}$ unconfirmed $(\mathrm{CRu})$ or in $\mathrm{PR}$ at the end of the chemoimmunotherapy program.

\section{Response Evaluation}

Intermediate response was assessed after the third course of R-CHOP21, and final response was evaluated after the end of chemoimmunotherapy and RT. CR, CRu and PR were defined according to Cheson's 1999 criteria. ${ }^{17}$ No response was defined as any response less than a PR, progressive disease, or death during the treatment period.

\section{Sample Size and Study End Points}

According to the evidence available when the study was planned, the sample size was calculated by using PFS as the primary end point. Given a 5 -year PFS of $48 \%$ reported in the previous IELSG retrospective analysis on 373 unselected patients with PTL, the sample size was calculated to show an improvement of $20 \%$ in the 5-year PFS for the new treatment policy. A one-arm trial that included 53 patients was required to reject the null hypothesis of a 5-year PFS of 50\% under the alternative hypothesis that the true 5-year PFS was $70 \%$, with a one-sided 5\% significance level and 80\% power, assuming a 4-year accrual and 2-year minimum follow-up.

OS, PFS, and time to progression (TTP) were calculated from the date of diagnosis and defined according to the revised National Cancer Institute (NCI) criteria. ${ }^{18}$ PFS includes all patients with an event defined as a progression at any time during treatment, less than a CR/CRu at the end of treatment, relapse, or death from any cause. OS includes all patients, with an event defined as the death of a patient due to any cause. TTP is defined as the time from study entry until documented lymphoma progression or death as a result of lymphoma.

\section{Statistical Methods}

Cumulative survival curves were generated by using the Kaplan-Meier method. ${ }^{19}$ Estimated percent of event rates were derived from the KaplanMeier estimates and reported with 95\% CIs. Incidence of CNS recurrences in the presence of death as a competing risk and incidence of lymphoma progression or death as a result of lymphoma (TTP) were estimated by using the cumulative incidence method. ${ }^{20}$ In TTP, deaths from other causes were treated as competing events. The follow-up was updated as of October 31, 2009. All calculations were performed by using the cmprsk package of $\mathrm{R}$ software, version 2.6.0 (R Development Core Team 2007).

\section{RESULTS}

\section{Patient Characteristics}

From June 2001 to December 2006, 53 consecutive patients were enrolled (Table 1). The median age was 64 years (range, 22 to 79 years); 40 patients had stage I and 13 had stage II disease. Four patients had bilateral testicular involvement at diagnosis. Lactate dehydrogenase or $\beta_{2}$-microglobulin levels above normal values were observed in six patients.

\section{Feasibility}

Fifty-two of the 53 patients (98\%) completed R-CHOP21 as planned, and the remaining patient experienced early progression after four courses (Fig 1). There were no major protocol deviations regarding chemoimmunotherapy; all patients received the scheduled number of courses according to stage and response. Prophylactic testicular RT was performed in 47 patients (89\%; 36 stage I and 11 stage II); six patients did not receive prophylactic testicular RT because of bilateral orchiectomy at diagnosis in one, disease progression in one, and refusal in four. In stage II patients, nine received retroperitoneal lymph node RT. Median delivered dose of testicular RT was $30 \mathrm{~Gy}$ (range, 24 to $40 \mathrm{~Gy}$ ), and median delivered dose of IF-RT to lymph nodes was $30 \mathrm{~Gy}$ (range, 23 to $45 \mathrm{~Gy}$ ).

CNS prophylaxis was completed as planned in 50 patients (94\%), but it was discontinued in three patients because of poor tolerance $(\mathrm{n}=2)$ and toxicity $(\mathrm{n}=1)$. These three patients received one, two, and three IT-MTX doses, respectively.

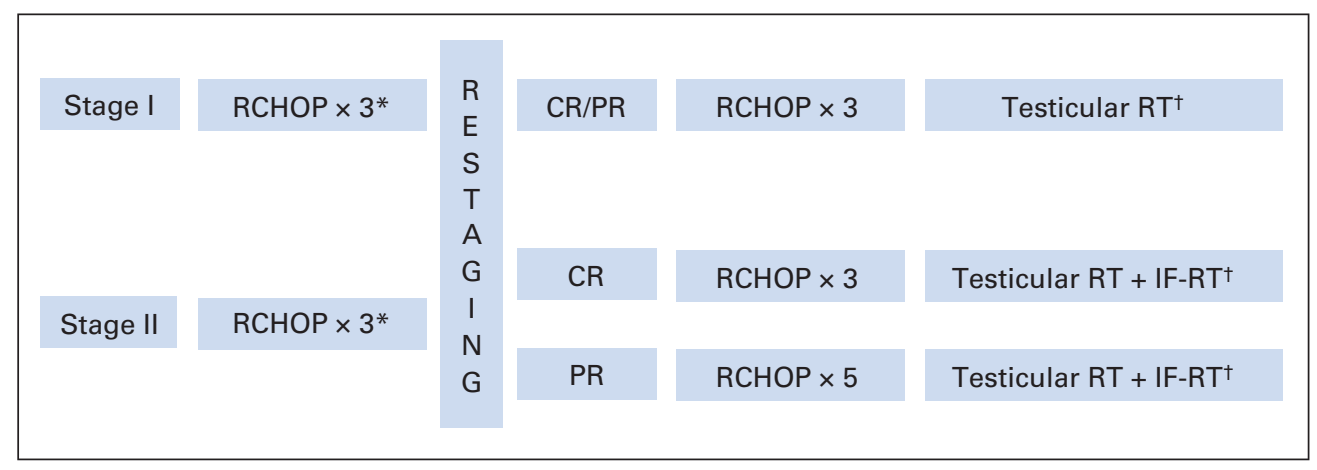

Fig 2. Plan of treatment for the International Extranodal Lymphoma Study Group 10 (IELSG-10) study. (*) CNS prophylaxis: $12 \mathrm{mg}$ intrathecal methotrexate during the first two courses of rituximab plus cyclophosphamide, doxorubicin, vincristine, and prednisone administered every 21 days (RCHOP21; days 1, 8, 15, 22). (†) Testicular radiotherapy (RT): prophylactic testicular irradiation including the contralateral testis at 25-30 Gy; involved field RT (IF-RT) at 30-35 Gy in stage II patients in complete response (CR) after RCHOP21, at 35-45 Gy in stage II patients in CR unconfirmed/partial response (PR) after RCHOP21. 


\section{Response and Outcome}

Fifty-two patients (98\%) achieved a CR, and only one experienced progressive disease at nodal and extranodal sites during treatment and died. At a median follow-up of 65 months, nine patients experienced relapse. Relapses involved lymph node alone in two patients, extranodal organs (pleura, skin, unknown) with or without lymph nodes in four patients, and CNS in three patients. Five of these patients died of lymphoma and four are currently alive in second remission after salvage therapy. Overall, treatment failures were observed in nine of 40 patients with stage I disease and in one of 13 patients with stage II disease. This last patient had both extranodal and nodal relapse involving pleura and retroperitoneal lymph nodes within the RT field (actual delivered dose, $38 \mathrm{~Gy}$ ).

With a median follow-up of 65 months, the 5-year PFS was $74 \%$ (95\% CI, 59\% to 84\%; Fig 3A). CNS relapses occurred in three patients: two of them had isolated CNS relapses (one meningeal and one brain parenchymal disease) and one had a concurrent meningeal and lymph nodal relapse. The two patients with isolated CNS relapse received prophylactic IT-MTX as planned by the protocol; the third patient received only two IT-MTX doses because of poor tolerance. The 5-year cumulative incidence of CNS relapse, taking into account the competitive risk of death, was $6 \%$ (95\% CI, $0 \%$ to $12 \%$; Fig 4 ). No contralateral testis relapses were observed.

The 5-year OS rate was $85 \%$ (95\% CI, $71 \%$ to $92 \%$; Fig 3B). Ten patients died: six of progressive disease, two of acute myelogenous leukemia after 21 and 60 months off therapy, one of heart failure, and one of gastric cancer after 17 and 9 months of follow-up. Overall, one patient progressed and nine relapsed, so the 5-year cumulative incidence of lymphoma progression or death as a result of lymphoma (TTP) was $18 \%$ (95\% CI, $7 \%$ to $29 \%$; Fig 3C).

\section{Toxicity and Safety}

Hematologic toxicity was mild during the R-CHOP21 treatment. According to the WHO toxicity criteria, a grade $\geq 3$ hematologic toxicity for neutrophils was recorded in 14 patients (26\%), platelets in four ( $8 \%$ ), and hemoglobin in two (4\%). Grade $\geq 3$ nonhematologic toxicities were reported in 13 patients (Table 2 ). Grade $\geq 3$ neurologic toxicity was observed in seven patients (13\%): two had sensory neuropathy and one had motor neuropathy due to vincristine; four patients had severe headache after IT-MTX of whom one had a cerebrospinal fluid leak and one a subdural hematoma. CNS prophylaxis was interrupted in three of them. Severe infections were reported in only two patients (4\%): one patient had a staphylococcus sepsis after the third course of R-CHOP21, and one had a bacterial pneumonia after the fourth course of R-CHOP21 and stopped chemotherapy while in disease progression. No deaths as a result of toxicity occurred during treatment. Fatal late adverse events, possibly associated with the treatment, occurred in three patients (6\%): two acute myelogenous leukemia and one heart failure (see causes of death in Table 3 ).

\section{DISCUSSION}

The IELSG-10 study is, to the best of our knowledge, the first worldwide prospective trial on PTL. This trial showed, in a cohort of patients with a prolonged follow-up, that a combined treatment with RCHOP21 associated with CNS and testicular prophylaxis is a promis-

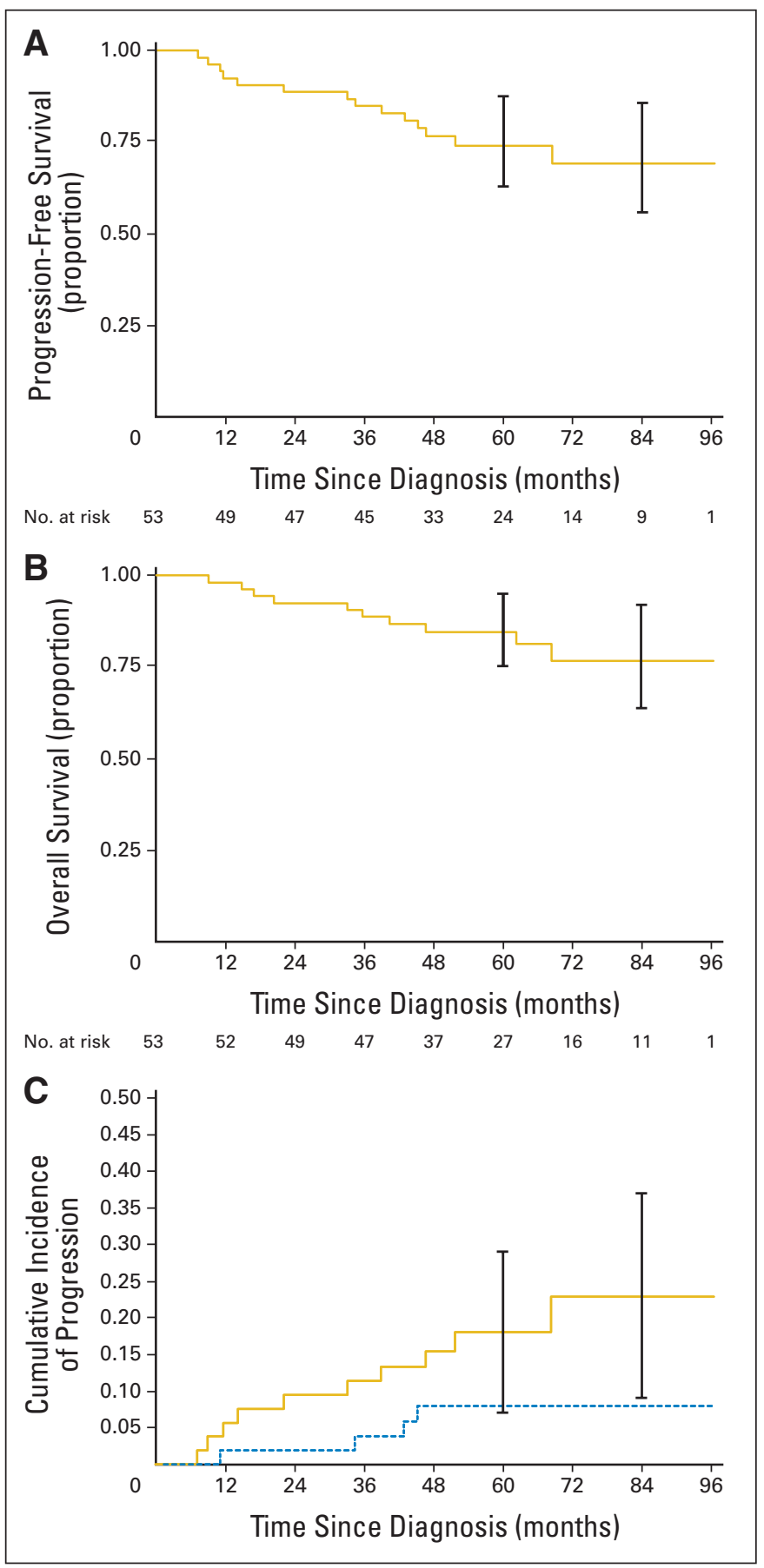

Fig 3. (A) Progression-free survival (PFS), (B) overall survival (OS), and (C) cumulative incidence of time to progression (TTP, solid gold line; cumulative mortality without progression, dashed blue line); 5-year PFS, 74\% (95\% Cl, 59\% to $84 \%)$; 5 -year OS, $85 \%(95 \% \mathrm{Cl}, 71 \%$ to $92 \%)$; and 5 -year TTP, $18 \%(95 \% \mathrm{Cl}$, $7 \%$ to $29 \%$ ). Vertical bars represent $95 \%$ Cls.

ing approach for PTL. This strategy is associated with a good outcome with 5-year PFS, OS, and cumulative incidence of TTP of $74 \%, 85 \%$, and $18 \%$, respectively. An effective systemic control of the disease with no contralateral testis relapses and a low incidence of CNS relapse was achieved in this trial. The acceptable toxicity profile of this treatment is remarkable considering that, as usual for PTL, half the patients were older than age 65 years. 


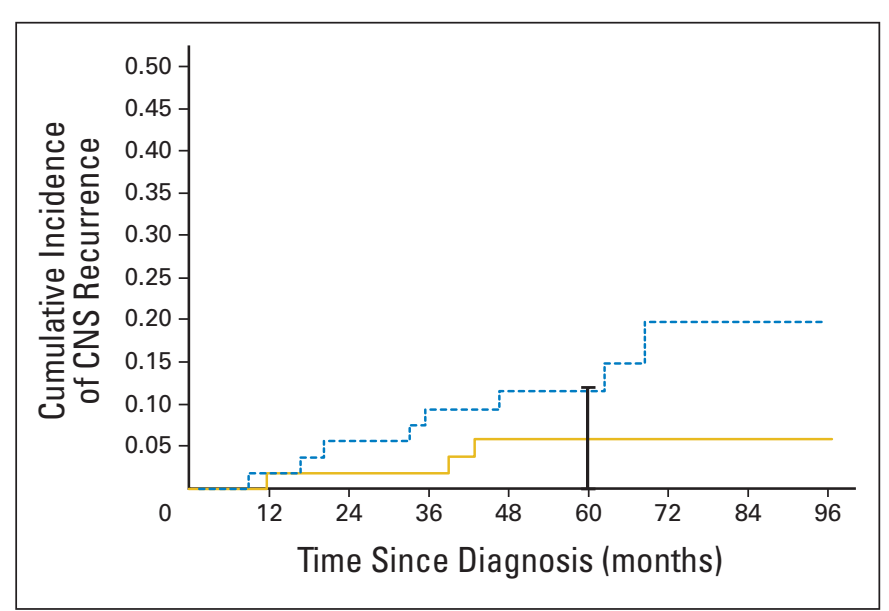

Fig 4. Cumulative incidence of CNS recurrence (solid gold line) and cumulative mortality without CNS involvement (dashed blue line); 5-year CNS cumulative incidence, $5.9 \%(95 \% \mathrm{Cl}, 0 \%$ to $12 \%)$. Vertical bar represents $95 \% \mathrm{Cl}$.

One limitation to our study should be noted. This is a phase II trial that includes a relatively small consecutive series of patients with PTL. Conversely, in a rare disease such as PTL, implementing a randomized phase III trial to make strong and definitive conclusions on efficacy is a complex issue, often unfeasible. Moreover, the lack of previous prospective studies on PTL led us to use, as best available reference, the 5-year PFS of a historical group of patients, which was not completely comparable with the group of patients enrolled on this phase II study. Thus, such a comparison should be viewed with caution.

A pattern of continuous relapses and disease-related deaths was shown in several studies in PTL series in localized stages. ${ }^{3,4,21,22}$ In the previous IELSG retrospective study, ${ }^{1}$ the PFS progressively declined at 5 and 10 years, from $48 \%$ to $33 \%$, respectively, in the whole series, and from $54 \%$ to $36 \%$ in the subset of stage I patients. A similar behavior was also observed in a recent population-based retrospective study ${ }^{4}$ with a 5- and 10-year disease-specific survival of $62 \%$ and $50 \%$, respectively (PFS was not reported). Indeed, we observed four late lymphoma-unrelated deaths in relapse-free patients.

Table 2. Summary of Acute Hematologic and Nonhematologic Toxicities

\begin{tabular}{|c|c|c|c|c|c|c|}
\hline \multirow[b]{3}{*}{ Acute Toxicity } & \multicolumn{6}{|c|}{ Grade } \\
\hline & \multicolumn{2}{|c|}{0} & \multicolumn{2}{|c|}{$1-2$} & \multicolumn{2}{|c|}{$3-4$} \\
\hline & No. & $\%$ & No. & $\%$ & No. & $\%$ \\
\hline Hematologic, total & 36 & 68 & 2 & 4 & 5 & 28 \\
\hline Anemia & 48 & 90 & 3 & 6 & 2 & 4 \\
\hline Neutropenia & 38 & 72 & 1 & 2 & 14 & 26 \\
\hline Thrombocytopenia & 49 & 92 & 0 & & 4 & 8 \\
\hline Neurologic & 39 & 74 & 7 & 13 & 7 & 13 \\
\hline Cardiac & 50 & 94 & 2 & 4 & 1 & 2 \\
\hline GI & 42 & 79 & 10 & 19 & 1 & 2 \\
\hline Infection & 48 & 90 & 3 & 6 & 2 & 4 \\
\hline Fever of unknown origin & 50 & 94 & 2 & 4 & 1 & 2 \\
\hline Cutaneous & 49 & 92 & 3 & 6 & 1 & 2 \\
\hline
\end{tabular}

\begin{tabular}{|lccccc|}
\hline \multicolumn{4}{|c|}{ Table 3. Late Possible Treatment-Associated Adverse Toxicities } \\
\hline & \multicolumn{5}{c}{$\begin{array}{c}\text { Time From } \\
\text { End of }\end{array}$} \\
Late Toxicity & $\begin{array}{c}\text { No. of } \\
\text { Patients }\end{array}$ & $\begin{array}{c}\text { Age } \\
\text { (years) }\end{array}$ & $\begin{array}{c}\text { Treatment } \\
\text { (months) }\end{array}$ & Outcome \\
\hline AML & 1 & 69 & 21 & Death \\
Myelodysplasia with AML & 1 & 79 & 44 & Death \\
Heart failure & 1 & 75 & 17 & Death \\
\hline Abbreviation: AML, acute myelogenous leukemia. & & \\
\hline
\end{tabular}

Notably, five of ten relapses were late events ( $>2$ years from the end of therapy), which is uncommon in most nodal and extranodal forms of DLBCL. ${ }^{23}$ This peculiar behavior of PTL underlines the necessity of prolonged follow-up in these patients. Accordingly, the median follow-up of this trial, which exceeds 5 years, may be not long enough to detect all of the events.

Our treatment included three components: prophylactic RT to the contralateral testis, addition of rituximab to CHOP, and CNS prophylaxis. In the previous retrospective IELSG study, ${ }^{1}$ prophylactic RT to the contralateral testis appeared to prevent testicular relapses, which were reduced to $8 \%$ compared with $35 \%$ in those not irradiated. Positive effect of prophylactic RT to the contralateral testis in this trial further supports the inclusion of this strategy into international clinical recommendations for PTL. ${ }^{24}$ In this study, the small number of patients with stage II disease precluded proper assessment of the role of adding RT to retroperitoneal lymph nodes. Indeed, the role of $\mathrm{RT}$ after R-CHOP is still controversial in nodal DLBCL. ${ }^{25}$

The addition of rituximab to CHOP chemotherapy may have improved outcome by preventing relapses with better control of microscopic systemic disease that might have been present since the onset of the disease. The benefit of the addition of rituximab in the treatment of PTL was recently questioned by the population-based retrospective study, which showed no difference in the outcome of patients with PTL treated before and after the rituximab era, using the year 2000 as the cut point. ${ }^{4}$ However, this retrospective study was limited by the lack of information regarding treatment delivered and what proportion of patients actually received rituximab-containing chemotherapy regimens. Although only randomized clinical trials can address the impact of rituximab in PTL, these studies are difficult to perform because of the rarity of this disease. Many studies of patients with predominantly nodal DLBCL have provided strong evidence of the benefit of the addition of rituximab to chemotherapy in DLBCL, which could be an indirect proof of a potential benefit also in PTL. ${ }^{13-15,26}$ Importantly, there is discordant published evidence on the role of rituximab in preventing CNS relapses in DLBCL. CNS recurrence rate was similar in 399 elderly DLBCL patients treated with $\mathrm{R}-\mathrm{CHOP}$ or $\mathrm{CHOP} .{ }^{27}$ Conversely, the analysis of CNS events in 1,222 elderly DLBCL patients treated in the RICOVER-60 trial showed that the addition of rituximab to CHOP14 is associated with a reduction of the relative risk of CNS disease to $0.58 .^{28}$ Although this question remains open, it is likely that a better systemic disease control in DLBCL patients treated with R-CHOP might also result in a reduced risk of CNS recurrences.

A retrospective series of 24 patients with PTL, treated before the rituximab era with a similar trimodality strategy (doxorubicin-based chemotherapy, testicular RT, and IT-MTX) has shown a 5-year PFS 
and OS of $78 \%$ and $66 \%$, respectively, but both without an apparent curve plateau. Moreover, the risk of CNS relapse was still high (16\%). ${ }^{29,30}$ A comparison of these results with those of the IELSG-10 study may suggest that there could be some benefit from the addition of rituximab. However, a longer follow-up is needed to clarify the role of rituximab in PTL treatment.

The incidence of CNS relapses in both brain parenchyma and meninges is more common in PTL than in other aggressive lymphomas. This complication is usually observed during the first 2 years of follow-up in nodal DLBCL, ${ }^{31}$ whereas late CNS relapses have been reported in PTL. ${ }^{1,6-10}$ In the previous IELSG series, 5- and 10-year risks of CNS relapse of $20 \%$ and $35 \%$ were observed. ${ }^{1}$ In this study, the cumulative incidence of CNS relapse at 5 years was only $6 \%$. However, in the absence of a comparison group without CNS prophylaxis, we cannot ascribe these results to the introduction of CNS prophylaxis. The best strategy for preventing CNS relapse is still a matter of debate. The value of prophylactic intrathecal chemotherapy is controversial because CNS relapses occur more frequently in brain parenchyma than in meninges and also in patients who have received intrathecal chemotherapy. ${ }^{9,14,16}$ However, PTL patients are usually elderly and many of them may not tolerate aggressive CNS prophylaxis such as systemic high-dose methotrexate or cytarabine chemotherapy regimens. The CNS prophylaxis chosen in our study-four doses of ITMTX - is easy to administer with good compliance and low toxicity despite the advanced age of the patients, and it was feasible in $94 \%$ of the patients. Nevertheless, in our study, despite CNS prophylaxis, three patients experienced CNS disease, and one of them had parenchymal disease. This underlines the necessity of further studies to properly define the best strategy for CNS prophylaxis in patients with PTL. For instance, the use of drugs with a higher CNS bioavailability, such as intrathecal liposomal cytarabine and/or the addition of systemic intermediate-dose methotrexate, may play a role in preventing CNS relapses. A new prospective study (IELSG-30) conducted by the IELSG is ongoing to test this hypothesis.

In conclusion, this study showed that R-CHOP21 combined with CNS and testicular prophylaxis is feasible in patients younger than age 80 years with PTL. However, the long-term benefit of this strategy should be confirmed with a longer follow-up in a larger series of patients. The results of this study might be considered as a reference for future studies on this rare lymphoma.

\section{AUTHORS' DISCLOSURES OF POTENTIAL CONFLICTS} OF INTEREST

Although all authors completed the disclosure declaration, the following author(s) indicated a financial or other interest that is relevant to the subject matter under consideration in this article. Certain relationships marked with a " $U$ " are those for which no compensation was received; those relationships marked with a "C" were compensated. For a detailed description of the disclosure categories, or for more information about ASCO's conflict of interest policy, please refer to the Author Disclosure Declaration and the Disclosures of Potential Conflicts of Interest section in Information for Contributors.

Employment or Leadership Position: None Consultant or Advisory Role: Umberto Vitolo, Roche Italy (C); Armando Lopez-Guillermo, Roche (C) Stock Ownership: None Honoraria: None Research Funding: None Expert Testimony: None Other Remuneration: None

\section{AUTHOR CONTRIBUTIONS}

Conception and design: Umberto Vitolo, Mary K. Gospodarowicz, Franco Cavalli, Andreas H. Sarris, Emanuele Zucca

Administrative support: Franco Cavalli, Emanuele Zucca

Provision of study materials or patients: Umberto Vitolo, Annalisa Chiappella, Andrés J.M. Ferreri, Maurizio Martelli, Monica Balzarotti, Chiara Bottelli, Annarita Conconi, Henry Gomez, Armando

Lopez-Guillermo, Giovanni Martinelli, Francesco Merli, Domenico Novero, Lorella Orsucci, Vincenzo Pavone, Umberto Ricardi, Sergio Storti, Emanuele Zucca

Collection and assembly of data: Umberto Vitolo, Annalisa Chiappella, Ileana Baldi, Emanuele Zucca

Data analysis and interpretation: Umberto Vitolo, Annalisa Chiappella, Andrés J.M. Ferreri, Ileana Baldi, Umberto Ricardi, Mary K.

Gospodarowicz, Franco Cavalli, Andreas H. Sarris, Emanuele Zucca

Manuscript writing: Umberto Vitolo, Annalisa Chiappella, Andrés J.M. Ferreri, Maurizio Martelli, Ileana Baldi, Monica Balzarotti, Chiara Bottelli, Annarita Conconi, Henry Gomez, Armando Lopez-Guillermo, Giovanni Martinelli, Francesco Merli, Domenico Novero, Lorella Orsucci, Vincenzo Pavone, Umberto Ricardi, Sergio Storti, Mary K. Gospodarowicz, Franco Cavalli, Andreas H. Sarris, Emanuele Zucca Final approval of manuscript: Umberto Vitolo, Annalisa Chiappella, Andrés J.M. Ferreri, Maurizio Martelli, Ileana Baldi, Monica Balzarotti, Chiara Bottelli, Annarita Conconi, Henry Gomez, Armando Lopez-Guillermo, Giovanni Martinelli, Francesco Merli, Domenico Novero, Lorella Orsucci, Vincenzo Pavone, Umberto Ricardi, Sergio Storti, Mary K. Gospodarowicz, Franco Cavalli, Andreas H. Sarris, Emanuele Zucca

\section{REFERENGES}

1. Zucca E, Conconi A, Mughal TI, et al: Patterns of outcome and prognostic factors in primary largecell lymphoma of the testis in a survey by the International Extranodal Lymphoma Study Group. J Clin Oncol 21:20-27, 2003

2. Shahab N, Doll DC: Testicular lymphoma. Semin Oncol 26:259-269, 1999

3. Møller MB, d'Amore F, Christensen BE: Testicular lymphoma: A population-based study of incidence, clinicopathological correlations and prognosis-The Danish Lymphoma Study Group, LYFO. Eur J Cancer 30A:1760-1764, 1994

4. Gundrum JD, Mathiason MA, Moore DB, et al: Primary testicular diffuse large b-cell lymphoma: A population-based study on the incidence, natural history, and survival comparison with primary nodal counterpart before and after the intro- duction of rituximab. J Clin Oncol 27:5227-5232, 2009

5. Booman M, Douwes J, Glas AM, et al: Primary testicular diffuse large B-cell lymphomas have activated B-cell-like subtype characteristics. J Pathol 210:163-171, 2006

6. Seymour JF, Solomon B, Wolf MM, et al: Primary large-cell non-Hodgkin's lymphoma of the testis: A retrospective analysis of patterns of failure and prognostic factors. Clin Lymphoma 2:109-115, 2001

7. Fonseca R, Habermann TM, Colgan JP, et al: Testicular lymphoma is associated with a high incidence of extranodal recurrence. Cancer 88:154-161, 2000

8. Crellin AM, Hudson $B V$, Bennett $M H$, et al: Non-Hodgkin's lymphoma of the testis. Radiother Oncol 27:99-106, 1993

9. Ferry JA, Harris NL, Young $\mathrm{RH}$, et al: Malignant lymphoma of the testis, epididymis, and sper- matic cord: A clinicopathologic study of 69 cases with immunophenotypic analysis. Am J Surg Pathol 18:376-390, 1994

10. Tondini C, Ferreri AJ, Siracusano L, et al: Diffuse large-cell lymphoma of the testis. J Clin Oncol 17:2854-2858, 1999

11. Touroutoglou N, Dimopoulos MA, Younes A, et al: Testicular lymphoma: Late relapses and poor outcome despite doxorubicin-based therapy. J Clin Oncol 13:1361-1367, 1995

12. Doll DC, Weiss RB: Malignant lymphoma of the testis. Am J Med 81:515-524, 1986

13. Coiffier B, Lepage $E$, Briere J, et al: CHOP chemotherapy plus rituximab compared with $\mathrm{CHOP}$ alone in elderly patients with diffuse large-B-cell lymphoma. N Engl J Med 346:235-242, 2002

14. Pfreundschuh $M$, Trümper $L$, Osterborg $A$, et al: CHOP-like chemotherapy plus rituximab versus CHOP-like chemotherapy alone in young patients with good-prognosis diffuse large-B-cell lymphoma: 
A randomised controlled trial by the MabThera International Trial (MInT) Group. Lancet Oncol 7:379-391, 2006

15. Pfreundschuh $M$, Schubert J, Ziepert M, et al: Six versus eight cycles of bi-weekly CHOP-14 with or without rituximab in elderly patients with aggressive CD20+ B-cell lymphomas: A randomised controlled trial (RICOVER-60). Lancet Oncol 9:105-116, 2008

16. Gatter KC, Warnke RA: Diffuse large B-cell lymphoma, in Jaffe ES, Harris NL, Stein H, et al (eds): World Health Organization Classification of Tumours. Pathology and Genetics of Tumours of Haematopoietic and Lymphoid Tissues. Lyon, France, IARC Press, 2001, pp 171-174

17. Cheson BD, Horning SJ, Coiffier B, et al: Report of an international workshop to standardize response criteria for non-Hodgkin's lymphomas: $\mathrm{NCl}$ Sponsored International Working Group. J Clin Oncol 17:1244, 1999

18. Cheson BD, Pfistner B, Juweid ME, et al: Revised response criteria for malignant lymphoma. J Clin Oncol 25:579-586, 2007

19. Kaplan EL, Meier P: Nonparametric estimation from incomplete observations. J Am Stat Assoc 53:457-481, 1958
20. Kim HT: Cumulative incidence in competing risks data and competing risks regression analysis. Clin Cancer Res 13:559-565, 2007

21. Vitolo U, Ferreri AJ, Zucca E: Primary testicular lymphoma. Crit Rev Oncol Hematol 65:183-189, 2008

22. Linassier $C$, Desablens $B$, Lefrancq $T$, et al: Stage I-IIE primary non-Hodgkin's lymphoma of the testis: Results of a prospective trial by the GOELAMS Study Group. Clin Lymphoma 3:167-172, 2002

23. Larouche JF, Berger F, Chassagne-Clément $C$, et al: Lymphoma recurrence 5 years or later following diffuse large B-cell lymphoma: Clinical characteristics and outcome. J Clin Oncol 28:20942100, 2010

24. Zelenetz A, Abramson JS, Advani $\mathrm{RH}$, et al: NCCN Clinical Practice Guidelines in Oncology: NonHodgkin's lymphomas. J Natl Compr Canc Netw 8:288-334, 2010

25. Pfreundschuh M: How I treat elderly patients with diffuse large B-cell lymphoma. Blood 116:51035110, 2010

26. Sehn LH, Donaldson J, Chhanabhai M, et al: Introduction of combined CHOP plus rituximab therapy dramatically improved outcome of diffuse large B-cell lymphoma in British Columbia. J Clin Oncol 23:5027-5033, 2005
27. Feugier $\mathrm{P}$, Virion JM, Tilly $\mathrm{H}$, et al: Incidence and risk factors for central nervous system occurrence in elderly patients with diffuse large-B-cell Iymphoma: Influence of rituximab. Ann Oncol 15: 129-133, 2004

28. Boehme V, Schmitz N, Zeynalova S, et al: CNS events in elderly patients with aggressive lymphoma treated with modern chemotherapy (CHOP14) with or without rituximab: An analysis of patients treated in the RICOVER-60 trial of the German High-Grade Non-Hodgkin Lymphoma Study Group (DSHNHL). Blood 113:3896-3902, 2009

29. Sarris AH, Vitolo U, Zucca E, et al: Prospective management of primary testicular lymphoma (PTL) with doxorubicin-based chemotherapy, prophylactic intrathecal (IT) methotrexate and radiotherapy (RT), but without rituximab: Results from IELSG. Blood 108, 2006 (abstr 2454)

30. Mazloom A, Fowler $N$, lyengar $P$, et al: Primary testicular diffuse large B-cell lymphoma, M.D. Anderson Cancer Center experience. Blood 114, 2009 (abstr 2697)

31. van Besien K, Gisselbrecht C, Pfreundschuh $M$, et al: Secondary lymphomas of the central nervous system: Risk, prophylaxis and treatment. Leuk Lymphoma 49:52-58, 2008 